\title{
In vivo manipulation of stem cells for adipose tissue repair/reconstruction
}

\section{Kotaro Yoshimura*, Hitomi Eto, Harunosuke Kato, Kentaro Doi \& Noriyuki Aoi}

\begin{abstract}
Many features of adipose stem/progenitor cells, including their physiological functions and localization, have been clarified in the past decade. Adipose tissue turns over very slowly, with perivascular progenitor cells differentiating into new adipocytes to replace dead adipocytes. A number of clinical trials using freshly isolated or cultured adipose-derived stromal cells containing adipose progenitor/stem cells are ongoing. Therapeutic use of adipose stem/progenitor cells has been shown to promote angiogenesis and adipose tissue regeneration. Identification of adipocyte-releasing factors upon apoptosis/necrosis would be a breakthrough and could lead to the next stage for adipose tissue regeneration. Activation of precursors in perichondrium and periosteum shows a dramatic neogenesis by simple injection and is an ideal example of in situ tissue engineering. The 'hit and catch' strategy using a mobilizer of bone-marrow stem/progenitor cells (hit) and attractants to lead the cells to proper homing into the target tissue (catch) may be the future of stem cell manipulation. Careful design of the microenvironment, cell delivery protocol to avoid unexpected behavior and induce maximal potential, and selection of target diseases, will be critical to the success of clinical applications of adipose-derived stromal cells.
\end{abstract}

Among the dozens of trillions of cells in our body, we lose over 20 billion cells a day, requiring constant renewal to stay alive. Soft tissues of the body have relatively little turnover and are well protected compared with epithelial tissues, such as skin [1]. For adipose tissue engineering/regeneration, it is critical to understand the physiological roles and functions of adiposeresident perivascular progenitor cells (adipose-derived stromal cells [ASCs], containing adipose progenitor/ stem cells); this population, working together with vascular endothelial cells (VECs) and stem/progenitor cells recruited from bone marrow, is a main player in adipose tissue regeneration (adipogenesis and angiogenesis) [2]. Adipose tissue atrophy associated with aging likely results from an agedependent decrease in the number of ASCs and consequent impairment of physiological turnover, as is commonly seen in other tissues and organs [3,4]. In general, tissue-resident stem cells can be exhausted by excessive or repeated activation of stem cells, as can happen in chronic inflammation or irradiation therapies. For regenerative therapies, tissue-resident and/or recruited stem cells must be manipulated according to the condition or microenvironments of each disease. The target tissue conditions must be precisely evaluated in advance for stem cell pool, growth factor pool, vascularity, tissue oxygen tension, mechanical force, and inflammatory or infectious conditions. Here, we will review the latest research on how to manipulate tissue-resident or bone marrow stem/progenitor cells, for repair/reconstruction of adipose and related tissues.

\section{ASCs \& adipose tissue repair/ remodeling/regeneration}

Adipose tissue has been considered an organ of energy storage, the largest endocrine organ, a soft-tissue filler and a cosmetically unnecessary
"Author for correspondence: Kotaro Yoshimura, Department of Plastic Surgery, University of Tokyo School of Medicine, 7-3-1, Hongo, Bunkyo-Ku, Tokyo 113-8655, Japan; Tel.: +81 358008 948; Fax: +81 358008 947; kotaro-yoshimura@umin.ac.jp tissuediscarded by liposuction. Adipocytes have a lifespan of up to 10 years [5]. ASCs are regarded as a potent tool for cell-based therapies [6,7], comparable with bone marrow-derived mesenchymal stem cells, because ASCs can be obtained in large numbers through a less invasive approach, liposuction. Research on adipose tissue remodeling after injury provides insight on basic mechanisms of adipose tissue regeneration. ASCs, as progenitors of adipose tissue, are involved in any type of adipose tissue remodeling, including developmental growth, hyperplasia in obesity, postinjury repair [8] or ischemia [9], and tissue expansion induced by mechanical forces [10]. These remodeling processes are in balance between adipocyte apoptosis/necrosis and adipogenesis, and are always accompanied by capillary remodeling. Each adipocyte is directly attached to a capillary, which is also easily damaged by injury or hypoxia [8]. ASCs act as the main players of adipogenesis and angiogenesis in the adipose repair process by not only differentiating into adipocytes or VECs but also releasing angiogenic growth factors $[7,11,12]$. 


\section{Adipose tissue stem $\&$ progenitor cells}

Adipose tissue has many cells other than adipocytes, although adipocytes constitute more than $90 \%$ of the tissue volume. Our rough estimate through flow cytometry and 2D and 3D histology is that $1 \mathrm{~g}$ of intact adipose tissue contains $1,000,000$ adipocytes, $1,000,000$ ASCs, $1,000,000$ VECs and $1,000,000$ other cells (e.g., adipose-resident macrophages and lymphocytes, pericytes and fibroblasts) [13,14]. ASCs can be identified as $\mathrm{CD} 31 / \mathrm{CD} 34^{+} / \mathrm{CD} 45 / \mathrm{CD} 90^{+} /$ CD105/CD146 cells, but they become $\mathrm{CD}_{105^{+}}$when plated [15], while VECs are identified as $\mathrm{CD} 31^{+} / \mathrm{CD} 34^{+} / \mathrm{CD} 45^{-}$cells. It was recently discovered in mice that adipocyte progenitor cells are present in adipose vasculature [16] and identified as $\mathrm{Lin}^{-} / \mathrm{Scal}^{+} / \mathrm{CD} 24^{+} / \mathrm{CD} 29^{+} / \mathrm{CD} 34^{+}$ cells [17], but the relationship between ASCs and vascular pericytes remains to be verified [18]. Some studies demonstrated a $\mathrm{CD} 34^{+}$cell population localized in the vascular wall (adventitia) that can differentiate into vessels [19], which may also be related to ASCs. Very recently, another new multipotent (or pluripotent) stem cell has been identified in human adipose tissue, labeled by SSEA-3 [20,21]; they are localized in the connective tissue near the vessels or between adipocytes, and are definitely different from ASCs. This suggests that there may be two distinct undifferentiated populations, adipose stem and progenitor cells. The former may be multipotent (or even pluripotent) 'master stem' cells located near (but outside of) larger vessels, which stay quiescent and are only activated upon emergency, while the latter are adipose 'progenitor' cells (ASCs) located in capillaries (perivascular) and take care of physiological turnover of the tissue.

\section{- Hematopoietic cells resident in adipose tissue $\&$ their function}

Stromal vascular fraction obtained through collagenase digestion of adipose tissue contains not only CD45 adipose tissue-derived cells but also hematopoietic cells, because the adipose tissue originally contains not only some

Figure 1 (Right). Cellular events in ischemic adipose tissue.

Severe ischemia (such as $\mathrm{pO}_{2}<15 \mathrm{~mm} \mathrm{Hg}$ ) induces adipocyte death within $24 \mathrm{~h}$. If the ischemia is prolonged, vascular endothelial cells start to die. By contrast, adipose stem/progenitor cells can stay alive for up to $72 \mathrm{~h}$, even under severe conditions. During the critical first $72 \mathrm{~h}$, ASCs are activated by cell death factors (not identified) and try to repair the damaged tissue by proliferation, migration and differentiation, collaborating with infiltrating cells. If ASCs also die due to prolonged severe conditions, the adipose tissue will not be regenerated and the dead space will be filled with fibrosis.

ACS: Adipose-derived stromal cell; $\mathrm{pO}_{2}$ : Partial oxygen tension; VEC: Vascular endothelial cell.

circulating blood but also adiposeresident macrophages and lymphocytes. Liposuction aspirates contain a substantial number of erythrocytes and circulating leukocytes derived from hemorrhage. ASCs can be extracted not only from the supernatant fatty portion but also from the infranatant fluid portion (crystalloid) of liposuction aspirates [13,15]. Most of the contaminated erythrocytes can be disrupted and removed with hypotonic processing [15].

The stromal vascular fraction contains resident macrophages $\left(\mathrm{CD}^{2} 4^{+} / \mathrm{CD} 45^{+} / \mathrm{CD}^{206} 6^{+}\right)$which can be discriminated from circulating monocytes $\left(\mathrm{CD} 14^{+} \mathrm{CD} 45^{+} \mathrm{CD} 206^{-}\right)$by expression of CD206. Recently, some reports have suggested that adiposeresident lymphocytes are also associated with chronic inflammation in obese adipose tissue, which leads to adipocyte dysfunction and insulin resistance [22]. Our recent study indicated a new subset of monocyte/macrophages $\left(\mathrm{CD} 34^{+} / \mathrm{CD} 45^{+} / \mathrm{CD} 14^{+}\right.$cells) in human adipose tissue; they have multipotency, including high adipogenic capacity, and are strongly suggested to be involved in adipose tissue remodeling/ repair together with ASCs and may be physiologically provided from bone marrow [Eto ET AL., UnPublished Data].

\section{Adipose tissue repair/} remodeling after ischemia

\section{$\&$ injury}

Adipose tissue has the highest (50-60 $\mathrm{mmHg}$ ) partial oxygen tension $\left(p \mathrm{O}_{2}\right)$ among organs; most organs showed 5-20 mmHg. The high $p \mathrm{O}_{2}$ of adipose tissue likely reflects high density of capillary network and possibly the low oxygen consumption rate of the tissue. It has recently been suggested that diabetic adipose tissue is relatively ischemic, with low-grade chronic inflammation, which causes adipose endocrine dysfunction, insulin resistance and metabolic syndrome [23]. In surgically induced ischemia of nonobese mice, adipose tissue degenerated and subsequently remodeled with proliferation of ASCs and VECs [9]. Adipocytes die first $(<24 \mathrm{~h})$ under severe ischemia and VECs and blood-derived cells start to die next, while M1 macrophages later infiltrate and are involved in phagocytosis of dead adipocytes (Figure 1). By contrast, ASCs can stay alive for up to 3 days even under severe ischemia; during those 3 days, they are activated and contribute to the adaptive repair process through adipogenesis and angiogenesis $[2,8,9]$. If ASCs and stem cells die, the tissue can no longer regenerate and this results in tissue necrosis, leading to tissue atrophy or scar formation.

It has been suggested that an apoptotic cell releases specific signals to activate stem/progenitor cells and provide a new cell to replace the dying cell; this is called 'compensatory proliferation' [24]. Upon the activation of ASCs after injury, there seem to be some critical soluble factors released from dying cells and/or damaged extracellular matrix (ECM), which mediate the compensatory proliferation of ASCs and recruitment of bone marrow-derived progenitor/ stem cells, leading to subsequent repair of the tissue. Damage-associated molecular pattern molecules, such as high-mobility group box 1 (HMGB1), 


\section{Ischemia \\ $\left(\mathrm{pO}_{2}=15 \mathrm{mmHg}\right)$}

Critical 3 days

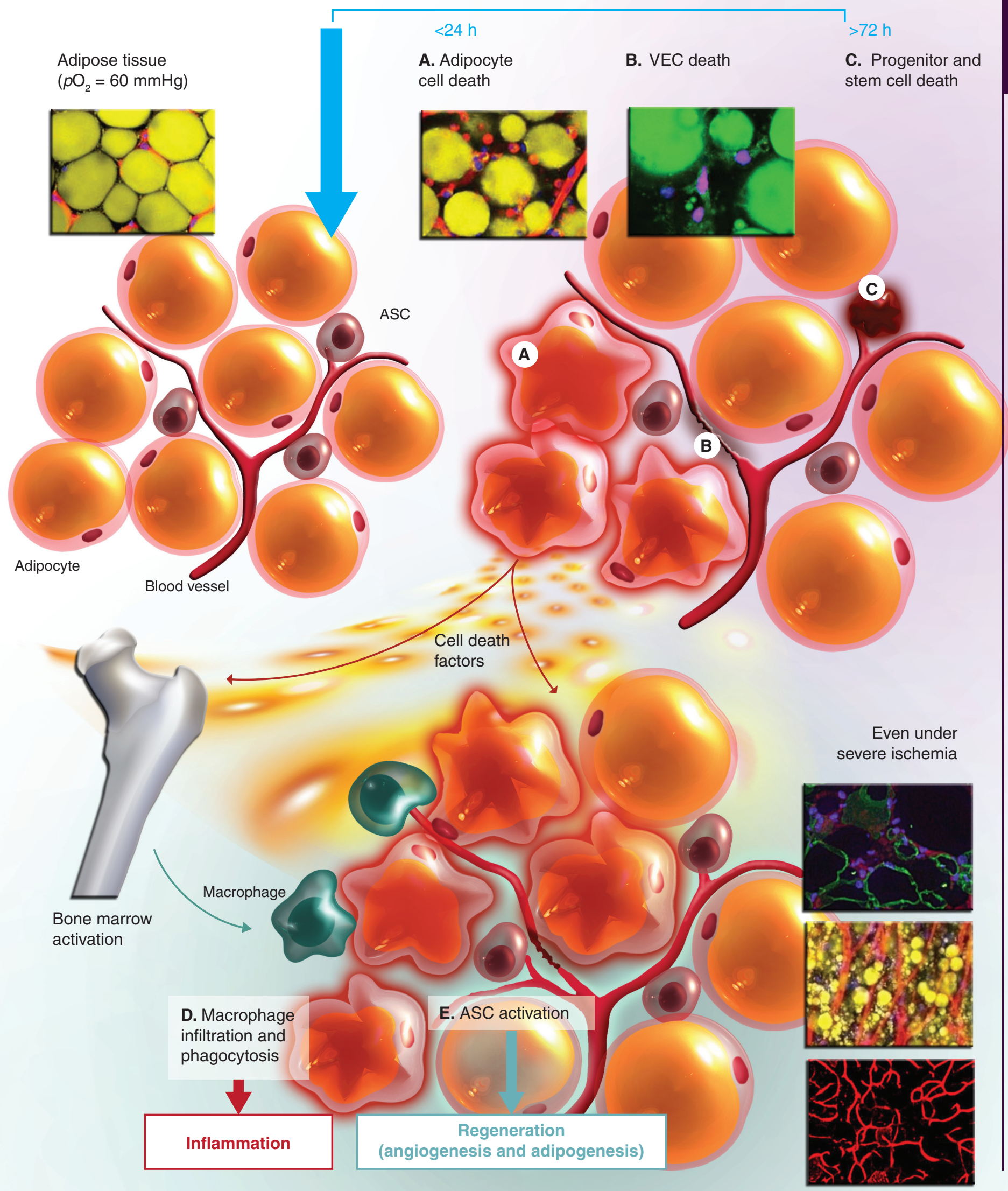


are released into the extracellular space and initiate wound healing by activating acute innate immunity and recruiting inflammatory cells $[25,26]$.

In an animal model of ischemiareperfusion injury to adipose tissue, basic FGF (bFGF) was locally released immediately after injury and stimulated ASCs not only to proliferate but also to release HGF [8]. In clinical injury to adipose tissue, a number of soluble factors are sequentially released in the injured site [27]. bFGF, PDGF, EGF and TGF- $\beta$ are released in the early (coagulation) phase (day 0-1) of wound healing, while VEGF, HGF, IL-8 and matrix metalloproteinase-1 gradually increase up to the late proliferation phase (day 5-7). On the other hand, KGF, IL-6 and matrix metalloproteinase- 8 peak during the inflammatory phase (day 2-4). Based on the results above, we prepared a growth factor mixture that we called adipose injury cocktail. Adipose injury cocktail promoted angiogenesis and improved oxygen tension in ischemic or diabetic adipose tissue by activating resident ASCs, suggesting that these factors may be beneficial for treating ischemic diseases [28].

\section{Adipose tissue repair/ remodeling after microfat grafting (lipofilling)}

Adipose tissue is used as an autologous filler for soft-tissue defects, but it has not been well documented how adipose grafts survive after nonvascularized transplantation (also called lipoinjection or lipofilling). In response to local hemorrhage from the injured recipient tissue, PDGF, EGF and TGF- $\beta$ are released from activated platelets [27]. The grafted adipose tissue is placed under severe ischemia (hypoxia with low nutrient) until direct vascular supply is formed [2]. In response to injury (adipocyte death, VEC death and ECM disruption), primary injury factors, such as bFGF, TNF- $\alpha$, TGF- $\beta$, damageassociated molecular pattern molecules and some proteases are likely released from the injured host tissue and dying grafted tissue (Figure 2) [2,29]. Recently, it was also demonstrated that EGF is released from apoptotic VECs and activates mesenchymal stem cells [30]. Inflammatory cells as well as endothelial progenitor cells are recruited from bone marrow to the injured tissue. ASCs are likely to be as resistant to ischemia as bone marrow-derived mesenchymal stem cells [31], stay functional for up to $72 \mathrm{~h}$ even under severe ischemia [9] and play pivotal roles in the repairing process.

Our recent study showed that almost all adipocytes located more than $300 \mu \mathrm{m}$ deep from the tissue surface die within a few days, although some are replaced little by little with next-generation adipocytes from the surface during the first 3 months. Surprisingly, we revealed that the partly necrotized adipose tissue does not lose its volume because dead adipocytes stay nonabsorbed as lipid droplets for a long time (several weeks to months depending on the diameter of the lipid droplet [32]). All lipid droplets are surrounded by infiltrating phagocytizing M1 macrophages [33]. If ASCs die owing to prolonged severe conditions (e.g., in the central area of the grafted tissue), the area is not properly replaced with new adipocytes, partly atrophies and is filled with fibrous scar tissue. Thus, grafted adipose tissue macroscopically looks like it has survived but the adipocytes are in fact mostly regenerated. Adipogenesis (indicated by new small adipocytes) was detected up to 3 months, while lipid droplet absorption was found to last up to 12 months. If a lipid droplet is huge, a cyst wall is formed before complete absorption and the oil cyst does not change size after the wall formation; the cyst wall starts to calcify over time. We summarize the postoperative cellular events in Figure 3 from adipocyte death on day 1 to oil cyst wall formation and calcification starting after 6 months. Differentiated cells die due to ischemia and undifferentiated cells survive and repair the tissue, but the tissue necrotize if the undifferentiated cells fail to survive; this finding on fat grafting seems to be common in any type of nonvascularized tissue grafting, such as skin graft.

Another study on exchanging fat graft between green fluorescent protein-expressing and wild-type mice revealed that most adipocytes and vascular structures seen at 4 weeks were derived from the donor animal, while many other cells, such as capillary endothelial cells and lipid dropletsurrounding cells (M1 macrophages), are derived from the host animal. The results suggested that adipose-resident stem/progenitor cells collaborated

\section{Figure 2 (Right). Cellular and molecular events after fat grafting.}

Adipose grafting induces injury in the recipient tissue; bleeding from the host tissue activates platelets, which release various soluble factors activating dormant stem/progenitor cells. At the same time, transplanted adipose tissue is temporarily placed under severe ischemia; primary factors, such as bFGF, are released from injured tissue or dying cells, stimulate mobilization of bone marrow cells and activate adipose-derived progenitor cells to release secondary growth factors, such as HGF, which promotes angiogenesis and inhibits fibrogenesis. Primary factors are released without transcriptional protein production, while secondary factors are produced and released by resident or recruited progenitor cells responding to the primary factors and other microenvironmental signals. Tissue injury further induces infiltration of other cells and release of inflammatory cytokines. The dead cells are partly replaced with next-generation cells derived from the stem/progenitor cells after successful vascularization.

CXCL: Chemokine (C-X-C motif) ligand; DAMP: Damage-associated molecular pattern molecule;

ECM: Extracellular matrix; EPC: Endothelial progenitor cell; MCP: Monocyte chemoattractant protein;

MSC: Mesenchymal stem cell; RANTES: Regulated on activation, normal T-cell expressed and secreted;

S1P: Sphingosine-1-phosphate; SDF-1: Stromal derived factor-1. 


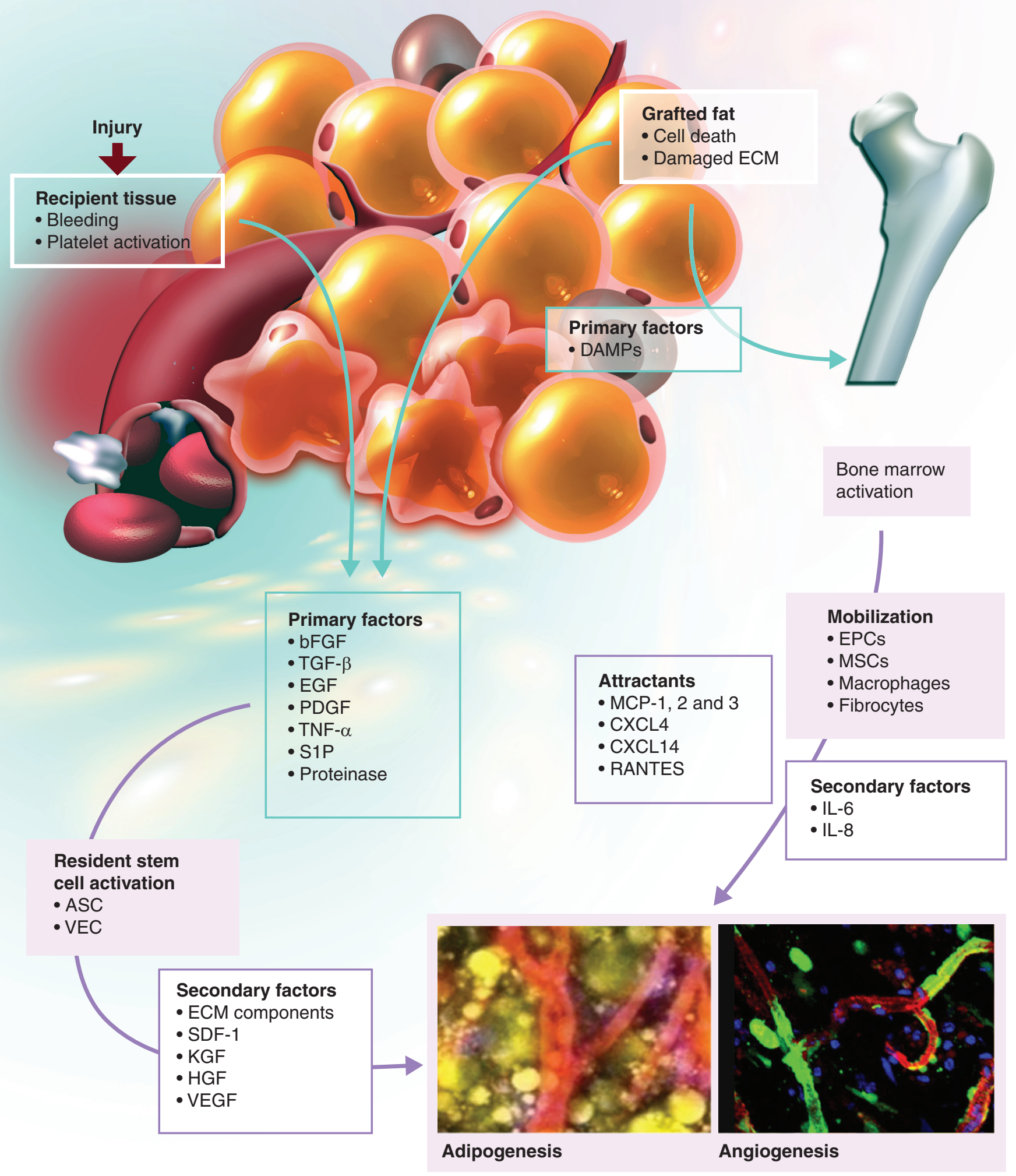

Regen. Med. ๑ Future Science Group (2011) 


\section{Postoperative sequence after fat grafting}

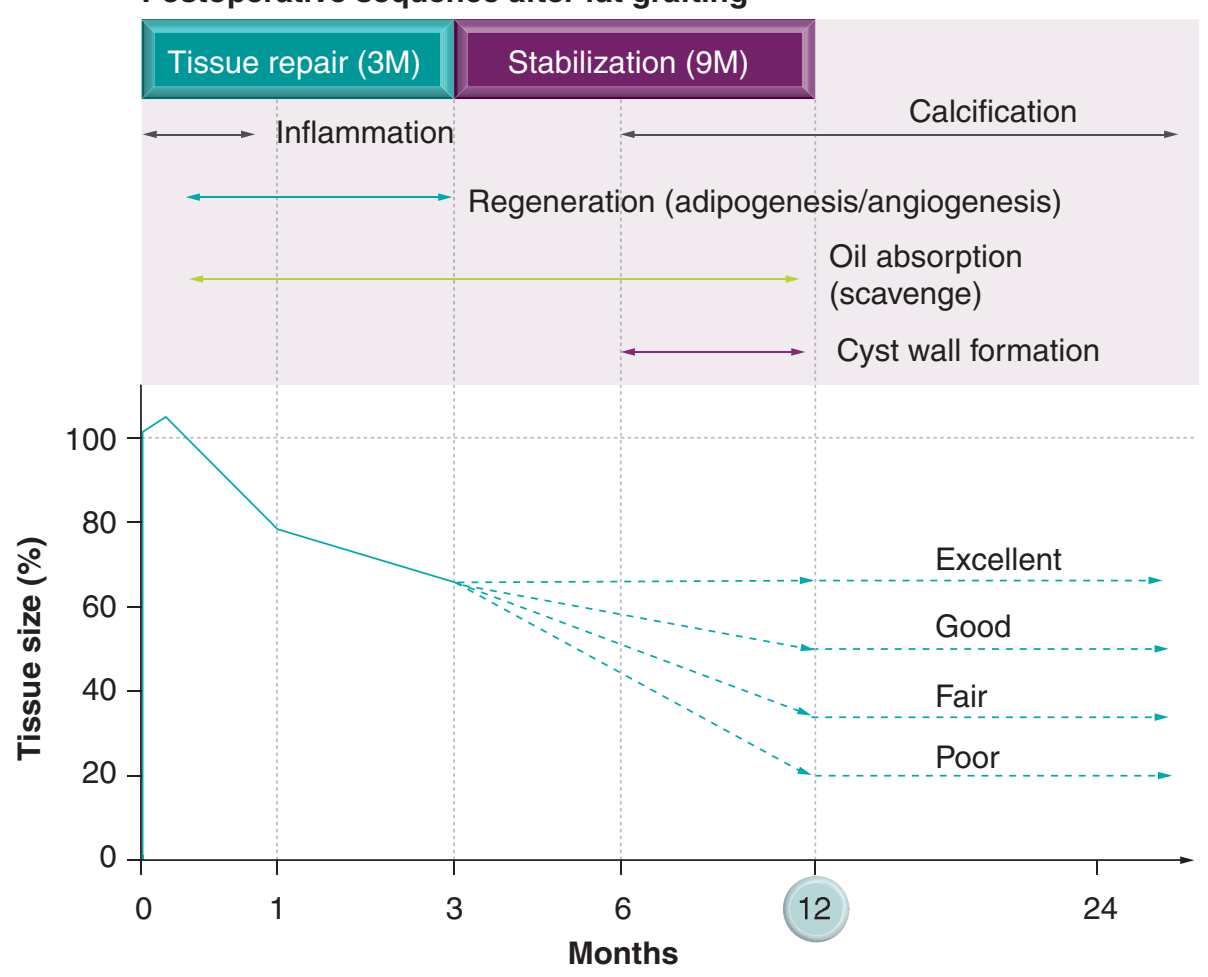

Figure 3. Long-term postoperative sequence after fat grafting.

Animal experiments of nonvascularized adipose tissue grafting indicated that almost all adipocytes (except for those located within $300 \mu \mathrm{m}$ from the tissue surface) die within a few days after adipose tissue grafting. Adipogenesis is exerted by activated adipose stem/progenitor cells and finishes by 3 months ('repair' phase), while remaining lipid droplets (dead adipocytes) are absorbed during the next 9 months ('stabilization' phase). Some of the dead adipocytes are replaced with new adipocytes of next generation during the first 3 months, while others are not. Lipid droplets are absorbed by macrophage phagocytosis, but the absorption is very slow and the absorption period is dependent on the diameter of the lipid droplets; when the lipid droplet diameter was large (e.g., $10 \mathrm{~mm}$ ), the cyst wall is formed before completing absorption and the cyst wall calcifies over time. In the central area of the graft, even adipose stem/progenitor cells die and new adipocytes are not produced, resulting in scar tissue or cyst formation. The final volume retention after adipose grafting is determined by the rate of successful replacement of adipocytes. If grafted, adipose has only small lipid droplets and absorption is finished by 3 months, the volume will not substantially change after 3 months (shown as 'excellent'). On the other hand, if many large lipid droplets remain at 3 months, tissue will atrophy between 3 and 12 months (shown as 'poor').

M: Months.

with other progenitor cells recruited from bone marrow in capillary reconstruction in the grafted fat tissue [Doi Ket al., Unpublished Data].

\section{- Innovations for better adipose tissue regeneration}

To overcome problems associated with fat grafting, such as unpredictable clinical results and a low rate of graft survival, many innovative efforts and refinements of surgical techniques have been reported. Condensation of living tissue and removal of unnecessary components have been performed by centrifugation, filtration or gravity sedimentation. External mechanical force has been used to expand the recipient tissue as well as the overlying skin envelop [10,34]. A recent experimental study suggested that repeated local injections of erythropoietin may enhance retention of grafted fat [35].

Stromal vascular fraction isolated from adipose or fluid portion of liposuction aspirates is supplemented to graft tissue to compensate its relative deficiency of ASCs (cell-assisted lipotransfer) [2,36-39]. This is based on our finding that aspirated fat tissue contains a much smaller number of ASCs (adipose progenitor cells) [40] and SSEA-3-positive stem cells (suspected to be master stem cells [20,21]) compared with intact fat tissue, probably because major vascular structures are not contained in aspirated fat tissue and many adipocytes and capillaries are ruptured by mechanical damage [13,14] . To use a dying cell (adipocyte) as a signal to activate ASCs appears to be a safe and reliable way to induce cell differentiation in the right direction (ASCs become the same cell type as the dying cell; e.g., adipocyte). If the dying tissue does not contain enough stem cells, the tissue regeneration may not be efficient. On the other hand, if only stem cells are transplanted, stem cells would not be sufficiently activated or differentiated due to lack of signals.

In our clinical trial, ectopic fibrogenesis and distant lymphadenopathy were observed in two patients [41]. In cell-based therapies using adherent mesenchymal stem cells, unfavorable cell behaviors, such as differentiation into myofibroblasts, have been reported [42]. In light of this, it was suggested that ASCs should be adhered to cells, tissue, ECM or biological scaffold before administration to avoid unexpected migration or differentiation.

\section{Stem cell manipulation for tissue repair/regeneration}

For therapeutic tissue (re) generation, stem cells and signals (microenvironments) are two critical 
requisites. Stem cells are actual players for tissue generation, maintenance and repair, while signals assign stem cells to specific fates. We need to control stem cell fate to avoid unexpected behavior of stem cells such as differentiation into unwanted direction [41,42], regardless of whether they are resident stem cells, recruited stem cells mobilized from bone marrow or administered stem cells.

The fate of ASCs, like any other stem cells, is determined by external signals, which include external stimuli such as adjacent cell death, ECM disruption, bleeding, inflammation, hypoxia, cytokines, chemokine, tissue injury and mechanical force. Many efforts have been made to identify specific signals useful for (re) generation of each organ, but there are few established so far for therapeutic uses. In the clinical setting, we can activate resident or bone marrow stem cells by wounding or in other ways, or can transplant stem cells or stem cell-containing tissue into stem cell-deficient, diseased or damaged organs with or without specific signals, although it is not easy to accurately manipulate the stem cells. Therapeutic manipulations of stem cells can be categorized into three concepts, described below.

\section{Stem cell activation with tissue wounding}

Quiescent stem cells can be activated by intentional tissue wounding, which is the simplest and most primitive method to activate resident stem cells for a therapeutic purpose. This approach has been commonly used for a long time in the field of plastic and dermatologic surgery. Skin resurfacing procedures such as chemical peeling, mechanical peeling and laser resurfacing are very popular for treating photoaged skin, not only its appearance but also skin functions [43]. By damaging or removing the tissue, resident stem cells are activated and then reconstruct/ renew the whole tissue in collaboration with infiltrated stem cells, which are recruited from the bone marrow [44]. For example, deep dermal peeling activates follicular stem cells and dermal fibroblasts, as well as bone marrow precursor cells, followed by renewal of the skin. Follicular stem cells in the hair bulge are activated upon abrasive skin wounding and can reconstruct the whole epidermis [45].

\section{Stem cell activation with nonwounding modalities}

There are many ways to activate resident stem cells other than tissue wounding. Growth factors can be topically applied or injected to the tissue; platelet-rich plasma is a cocktail of platelet-derived factors released upon aggregation of platelets and clinically used to promote or activate angiogenesis and tissue remodeling [46]. Bioactive materials such as a lattice made of ECM components and acellular dermal matrix can be applied to the raw surface or dead space of the tissue to promote wound healing, tissue contraction and epithelialization. Internal or external mechanical forces can be applied to the tissue; the skin and subcutaneous tissue can be easily expanded by an implanted tissue expander or external negative pressure $[10,34]$.

There are several clinical examples of natural tissue neogenesis, although they are not intentionally generated. Cauliflower ear (wrestler's ear) is a result of chondroneogenesis after hematoma between the auricular cartilage and skin, while osteogenesis is frequently seen at the edge of a tissue expander after scalp expansion [47]. In these cases, new tissue is generated between the perichondrium and cartilage or the periosteum and bone, respectively. It is strongly suggested that the detachment of periosteum from the bone activates resident stem cells in the periosteum and initiates osteogenesis in the dead space.

This brand new concept of tissue engineering can be applied to therapeutic tissue genesis. An injection of soft-tissue filler is the most frequently performed cosmetic procedure for facial rejuvenation, contouring and volumization. Most of the injectable materials, such as hyaluronic acid, are absorbable or degradable, and this is the major reason why their implantation is regarded as a safe procedure. These injectable materials can be injected under or around the periosteum or perichondrium to activate resident stem cells; the injectable is absorbed during several months, but the space is partly replaced with generated bone or cartilage, respectively. Thus, permanent tissue augmentation can be obtained by a minimally invasive treatment and this is a good example of in situ tissue engineering.

\section{Stem cell augmentation/ replacement therapies for stem cell-deficient tissues}

If the tissue is deficient in resident stem cells, the tissue will not heal quickly and may atrophy with time. Irradiated tissue is ischemic and fibrous, presumably as a result of stem cell deficiency. Chronic inflammation and autoimmune disease induce general fibrosis, presumably as a result of repeated activation and exhaustion of resident stem cells. Chronic wounds or ulcers are another example in this category. Generally, aged or photoaged tissues are related to atrophy and low repairing capacity resulting from a relative deficiency in stem cell number and functionality $[3,4]$. It could be a reasonable and effective resolution to transplant adipose tissue that contains sufficient stem cells into such stem cell-deficient tissue. Wound healing in irradiated tissue was shown to be improved by administration of adipose tissue or stem cells.

\section{Future perspectives}

A number of clinical trials using freshly isolated or cultured ASCs are ongoing in more than ten countries. Given the physiological functions of ASCs, its 


\section{Key points}

- Adipose tissue turns over very slowly (up to 10 years) and adipocyte progenitor cells are present in the vasculature of adipose tissue.

- Adipose tissue contains a substantial number (greater than adipocytes in number) of stromal cells, called adipose-derived stromal cells (ASCs). Some ASCs are multipotent and can differentiate into various lineages.

- ASCs divide, migrate and differentiate into adipocytes or vascular cells during the turnover/remodeling process that adipose tissue undergoes to compensate for apoptotic or degenerative changes. Every adipocyte needs direct contact with a capillary, thus adipogenesis is always accompanied by capillary angiogenesis.

- Microfat grafting is a promising method of soft-tissue augmentation due to the absence of both foreign materials and conspicuous scarring. There are still some issues to be resolved, including unpredictable effectiveness and long-term atrophy of grafted fat tissue.

- Aspirated fat tissue, the material used in microfat grafting, has fewer adipose progenitor cells than intact fat tissue. This may impair remodeling of the grafted tissue and lead to long-term atrophy of the grafts.

- Preliminary results from our clinical trials using progenitor-enriched fat tissue grafting suggested supplementation with vascular stromal fractions, containing adipose progenitor cells, may boost the efficacy and safety of conventional autologous microfat grafting.

- ASCs are a promising adult stem/progenitor cell population for use as a therapeutic tool. A therapeutic number of cells can be obtained without cell culture and the anticipated therapeutic potential is similar to marrow-derived mesenchymal stem cells.

\section{Bibliography}

Papers of special note have been highlighted as:

- of interest

1 Fuchs E. The tortoise and the hair: slow-cycling cells in the stem cell race. Cell 137(5), 811-819 (2009).

2 Yoshimura K, Suga H, Eto H. Adiposederived stem/progenitor cells: roles in adipose tissue remodeling and potential use for soft tissue augmentation. Regen. Med. 4(2), 265-274 (2009).

3 Caplan AI. Adult mesenchymal stem cells for tissue engineering versus regenerative medicine. J. Cell. Physiol. 213(2), 341-347 (2007).

4 Gago N, Pérez-López V, Sanz-Jaka JP et al. Age-dependent depletion of human skin-derived progenitor cells. Stem Cells 27, 1164-1172 (2009).

5 Spalding KL, Arner E, Westermark PO et al. Dynamics of fat cell turnover in humans. Nature 453(7196), 783-787 (2008).

- Approximately $10 \%$ of adipocytes are renewed annually in adults.

6 Zuk PA, Zhu M, Ashjian P et al. Human adipose tissue is a source of multipotent stem cells. Mol. Biol. Cell 13(12), 4279-4295 (2002).
7 Gimble JM, Katz AJ, Bunnell BA. Adiposederived stem cells for regenerative medicine. Circ. Res. 100(9), 1249-1260 (2007).

8 Suga H, Eto H, Shigeura T et al. IFATS collection: FGF-2-induced HGF secretion by adipose-derived stromal cells inhibits post-injury fibrogenesis through a JNKdependent mechanism. Stem Cells 27(1), 238-249 (2009).

9 Suga $\mathrm{H}$, Eto $\mathrm{H}$, Aoi $\mathrm{N}$ et al. Adipose tissue remodeling under ischemia: death of adipocytes and activation of stem/ progenitor cells. Plast. Reconstr. Surg. 126(6), 1911-1923 (2010).

10 Kato H, Suga H, Eto H et al. Reversible adipose tissue enlargement induced by external tissue suspension: possible contribution of basic fibroblast growth factor for preservation of enlarged tissue. Tissue Eng. Part A 16(6), 2029-2040 (2010).

11 Rehman J, Traktuev D, Li J et al. Secretion of angiogenic and antiapoptotic factors by human adipose stromal cells. Circulation 109(10), 1292-1298 (2004).

12 Miranville A, Heeschen C, Sengenes C et al. Improvement of postnatal neovascularization by human adipose tissue-derived stem cells. Circulation 110 (3), 349-355 (2004). homologous use, namely promoting adipogenesis/angiogenesis, would be the easiest and most promising way to perform ASC-based therapy. Careful design of microenvironments that activates ASCs, cell delivery protocol to avoid unexpected behavior and induce maximal potential of ASCs, and proper selection of target diseases, will be critical to the success of clinical applications.

\section{Financial \& competing}

interests disclosure

The authors have no relevant affiliations or financial involvement with any organization or entity with a financial interest in or financial conflict with the subject matter or materials discussed in the manuscript. This includes employment, consultancies, honoraria, stock ownership or options, expert testimony, grants or patents received or pending, or royalties.

No writing assistance was utilized in the production of this manuscript.

13 Suga H, Matsumoto D, Inoue K et al. Numerical measurement of viable and nonviable adipocytes and other cellular components in aspirated fat tissue. Plast. Reconstr. Surg. 122(1), 103-114 (2008).

14 Eto H, Suga H, Matsumoto D et al. Characterization of adipose tissue structure and cellular components: differences between aspirated adipose tissue and excised adipose tissue. Plast. Reconstr. Surg. 124(4), 1087-1097 (2009).

15 Yoshimura K, Shigeura T, Matsumoto D et al. Characterization of freshly isolated and cultured cells derived from the fatty and fluid portions of liposuction aspirates. J. Cell. Physiol. 208(1), 64-76 (2006).

16 Tang W, Zeve D, Suh JM et al. White fat progenitor cells reside in the adipose vasculature. Science 322(5901), 583-586 (2008).

\section{- Adipocyte progenitor cells reside in the} mural cell compartment of the adipose vasculature.

17 Rodeheffer MS, Birsoy K, Friedman JM. Identification of white adipocyte progenitor cells in vivo. Cell 135(2), 240-249 (2008).

18 Lin G, Garcia M, Ning H et al. Defining stem and progenitor cells within adipose tissue. Stem Cells Dev. 17(6), 1053-1063 
(2008).

19 Zengin E, Chalajour F, Gehling UM et al. Vascular wall resident progenitor cells: a source for postnatal vasculogenesis. Development 133(8), 1543-1551 (2006).

- Vascular progenitor cells exist in the adventitia of vessel walls.

20 Kuroda Y, Kitada M, Wakao S et al. Unique multipotent cells in adult human mesenchymal cell populations. Proc. Natl Acad. Sci. USA 107(19), 8639-8643 (2010).

- SSEA-3 $+/$ CD $105^{+}$multipotent (pluripotent) stem cells exist in various tissues and can be properly expanded only by a floating culture.

21 Wakao S, Kitada M, Kuroda Y et al. Multilineage-differentiating stressenduring (Muse) cells are a primary source of induced pluripotent stem cells in human fibroblasts. Proc. Natl Acad. Sci. USA 108(24), 9875-9880 (2011).

22 Nishimura S, Manabe I, Nagasaki M et al. $\mathrm{CD} 8{ }^{+}$effector $\mathrm{T}$ cells contribute to macrophage recruitment and adipose tissue inflammation in obesity. Nat. Med. 15(8), 914-920 (2009).

23 Pasarica M, Sereda OR, Redman LM et al. Reduced adipose tissue oxygenation in human obesity: evidence for rarefaction, macrophage chemotaxis, and inflammation without an angiogenic response. Diabetes 58(3), 718-725 (2009).

24 Fan Y, Bergmann A. Apoptosis-induced compensatory proliferation. The cell is dead. Long live the cell! Trends Cell Biol. 18(10), 467-473 (2008).

- Apoptosis induces activation of progenitor cells to replace the dead cell with a new cell: compensatory proliferation.

25 Lotze MT, Zeh HJ, Rubartelli A et al. The grateful dead: damage-associated molecular pattern molecules and reduction/oxidation regulate immunity. Immunol. Rev. 220, 60-81 (2007).

26 Tamai K, Yamazaki T, Chino $\mathrm{T}$ et al. PDGFR $\alpha$-positive cells in bone marrow are mobilized by high mobility group box 1 (HMGB1) to regenerate injured epithelia. Proc. Natl Acad. Sci. USA 108(16), 6609-6614 (2011).

27 Aiba-Kojima E, Tsuno NH, Inoue K et al. Characterization of wound drainage fluids as a source of soluble factors associated with wound healing: comparison with plateletrich plasma and potential use in cell culture. Wound Repair Regen. 15(4), 511-520 (2007).

Eto $\mathrm{H}$, Suga $\mathrm{H}$, Aoi $\mathrm{N}$ et al. Adipose injuryassociated factors activate adipose stem/ stromal cells, induce neoangiogenesis, and mitigate hypoxia in ischemic tissues. Am. J. Pathol. 178(5), 2322-2332 (2011).

29 Muthukrishnan L, Warder E, McNeil PL. Basic fibroblast growth factor is efficiently released from a cytolsolic storage site through plasma membrane disruptions of endothelial cells. J. Cell. Physiol. 148(1), 1-16 (1991).

30 Soulez M, Sirois I, Brassard N et al. Epidermal growth factor and perlecan fragments produced by apoptotic endothelial cells co-ordinately activate ERK1/2dependent antiapoptotic pathways in mesenchymal stem cells. Stem Cells 28(4), 810-820 (2010).

31 Myolotte LA, Duffy AM, Murphy M et al. Metabolic flexibility permits mesenchymal stem cell survival in an ischemic environment. Stem Cells 26(5), 1325-1336 (2008).

32 Pajvani UB, Trujillo ME, Combs TP et al. Fat apoptosis through targeted activation of caspase 8: a new mouse model of inducible and reversible lipoatrophy. Nat. Med. 11(7), 797-803 (2005).

33 Lumeng CN, Saltiel AR. Inflammatory links between obesity and metabolic disease. J. Clin. Invest. 121(6), 2111-2117 (2011).

34 Khouri R, Del Vecchio D. Breast reconstruction and augmentation using pre-expansion and autologous fat. Transplantation Clin. Plast. Surg. 36(2), 269-280 (2009).

35 Hamed S, Egozi D, Kruchevsky D et al. Erythropoietin improves the survival of fat tissue after its transplantation in nude mice. PLoS ONE 5(11), e13986 (2010).

36 Yoshimura K, Sato K, Aoi N, Kurita M, Hirohi T, Harii K. Cell-assisted lipotransfer (CAL) for cosmetic breast augmentation supportive use of adipose-derived stem/ stromal cells. Aesthetic Plast. Surg. 32(1), 48-55 (2008).

37 Yoshimura K, Sato K, Aoi N et al. Cellassisted lipotransfer for facial lipoatrophy: efficacy of clinical use of adipose-derived stem cells. Dermatol. Surg. 34(9), 1178-1185 (2008).

38 Yoshimura K, Asano Y, Aoi N et al.
Progenitor-enriched adipose tissue transplantation as rescue for breast implant complications. Breast J. 16(2), 169-175 (2010).

39 Yoshimura K, Asano Y. Fat injection to the breasts: cosmetic augmentation, implant replacement, inborn deformity, and reconstruction after mastectomy. In: Aesthetic and Reconstructive Surgery of the Breast. Hall-Findlay EJ, Evans GRD (Eds), Elsevier Ltd, London, UK, 405-420 (2010).

40 Matsumoto D, Sato K, Gonda K et al. Cell-assisted lipotransfer: supportive use of human adipose-derived cells for soft tissue augmentation with lipoinjection. Tissue Eng. 12(12), 3375-3382 (2006).

41 Yoshimura K, Aoi N, Suga H et al. Ectopic fibrogenesis induced by transplantation of adipose-derived progenitor cell suspension immediately after lipoinjection. Transplantation 85(12), 1868-1869 (2008).

42 Russo FP, Alison MR, Bigger BW et al. The bone marrow functionally contributes to liver fibrosis. Gastroenterology 130(6), 1807-1821 (2006).

43 Matarasso SL, Hanke CW, Alster TS. Cutaneous resurfacing. Dermatol. Clin. 15(4), 569-582 (1997).

44 Wu Y, Zhao RC, Tredget EE. Concise review: bone marrow-derived stem/ progenitor cells in cutaneous repair and regeneration. Stem Cells 28(5), 905-915 (2010).

45 Ito M, Liu Y, Yang Z et al. Stem cells in the hair follicle bulge contribute to wound repair but not to homeostasis of the epidermis. Nat. Med. 11(12), 1351-1354 (2005).

46 Eppley BL, Woodell JE, Higgins J. Platelet quantification and growth factor analysis from platelet-rich plasma: implications for wound healing. Plast. Reconstr. Surg. 114(6), 1502-1508 (2004).

47 Abrahamsson P, Isaksson S, Gordh $\mathrm{M}$ et al. Periosteal expansion of rabbit mandible with an osmotic self-inflatable expander. Scand. J. Plast. Reconstr. Surg. Hand Surg. 43(3), 121-125 (2009). 